\title{
Early Management of Open Tibial Fractures in Benin - City -Result of a Structured Treatment Protocol
}

\author{
Umebese Philipa ; Moin Mobash ; West Osemogie
}

\begin{abstract}
Open fractures of the tibia shaft are caused by major musculoskeletal injury, most times high energy transfer trauma. The determinants of a successful treatment outcome are effective infection control, skin coverage and adequate stabilization. We evaluated the outcome of a structured management protocol based on the afore mentioned determinants carried out on 39 patients with 39 open tibial shaft fractures. The fractures were categorized as type I - IIIC based on Gustillo Anderson Classification. There were 2 (5.1\%) type I, 17 (43.6\%) type II, 9

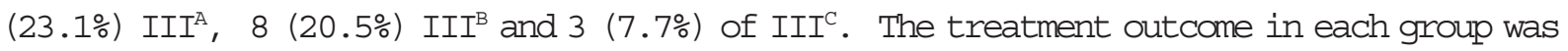
evaluated on the basis of primary wound healing and fracture union in line with the method of treatment chosen in the protocol.

Overall, primary wound closure was done in 17 (43.6\%) in types I, II and III with a $100 \%, 60 \%$ and $22 \%$ primary wound healing respectively. $15.4 \%$ wounds in type II and III (4 and 2 respectively) treated by delayed (primary) suturing proceeded to heal primarily. The success or healing rate with primary wound closure for types I \& II was significantly high $\left(\mathrm{x}^{2} .8 .743\right.$, of $\left.2 \mathrm{P} .0 .013\right) \mathrm{P}<0.05$ when compared with the overall outcome of cases treated with primary closure in other groups.

Of 11 cases in type III ${ }^{\mathrm{B}}$ and IIIC (8 and 3 respectively) who had delayed split thickness skin graft or fascio-cutanous flaps as primary procedure, 50\% had successful graft take.

Plaster of Paris was successfully used in 12 patients (30\%) with predominantly type I \& II fractures. Primary intramedullary nailing was carried out in 13 (33.3\%) cases out of which 69\% proceeded to union.
\end{abstract}

\section{INTRODUCTION}

Treatment of open fractures by early stabilization (internal/external) after debride-ment is a controversial subject. It runs counter to traditional approach and teaching of treating

KeY WoRDS: Open Fractures; Tibia; Early Management; Treatment Protocol.

*Orthopaedic \& Trauma Unit Dept of Surgery University of Benin Teaching Hospital Benin - City Nigeria.

Corresponding Author: Dr. Philip F.A. Umebese Dept of Surgery UBTH Benin - City Email: apexade@yahoo.com

(๑) CMS UNIBEN JMBR 2006; $5(2): 33-39$ soft tissues first and dealing with the bone secondarily $y^{1,2}$. Often in developing countries like Nigeria the cautionary aphorism of treating open fracture open is often ignored in most emergency rooms either because of non-availability or paucity of sterile materials for regular wound care. Sometimes the added cost of hospitalisation needed for regular dressings to render the wound clean and safe for eventual closure may be unaffordable to the patient. Hence, primary suturing becomes an attractively regular practice. Open fractures expose the fractures to infection at bed site, 
strip the bone of varying degrees of soft tissue coverage. Consequently, the denuded fragments may suffer devascularization and this may not be obvious or appreciated at the time of primary closure. A review of literature had shown that the prognosis associated with open fractures due to these profound complication, can be reduced if strict management guideline or protocol is followed because the meticulous care of the associated soft tissue injury with tibial fractures is of great importance and indeed critical to ideal treatment or good prognosis ${ }^{3,4,5}$. Gustillo \& Anderson (G-A) described different grades or types of open fractures with consideration of various degrees of soft tissue involvement. Other workers ${ }^{6}$ particularly Byrd in order to give a prognostic index to open fractures classified the bone and soft tissue lesion on basis of direct and indirect traumatic causative forces.

In order to give guidance to our casualty officers and orthopaedic residents on the appropriate and cost effective protocol for treating open fractures, we evaluated a structured treatment protocol for the 5 groups or types based on G-A open fractures classification. Our aim was to evaluate the treatment outcome of this structured protocol in terms of soft tissue injury healing and effective fracture stabilization and its suitability for adoption.

\section{Patients \& Methods}

This is a hospital based prospective study done in University of Benin Teaching Hospital Benin City, Nigeria. The patients were recruited and treated over a 12 month period (March 2002 to April 2003) and followed up for 2 years. All patients with extra articular open fractures of the shaft of the tibia with or without fibular fractures were included provided the initial management was done within 12 hours in the Accident \& Emergency (A\&E) wing of the hospital.

A total of 41 consecutive patients fulfilled the inclusion criteria and were entered initially. Two patients however, took their discharge against medical advice and so were excluded, leaving 39 patients in the study.

Their ages ranged from 20years to 85years with a mean age of $37.1\left(\mathrm{SD}_{ \pm 13.4)}\right.$ (Fig 2) . 92.5\% of the patients were male and all but 5 of the injuries resulted from vehicular accidents (Figl).

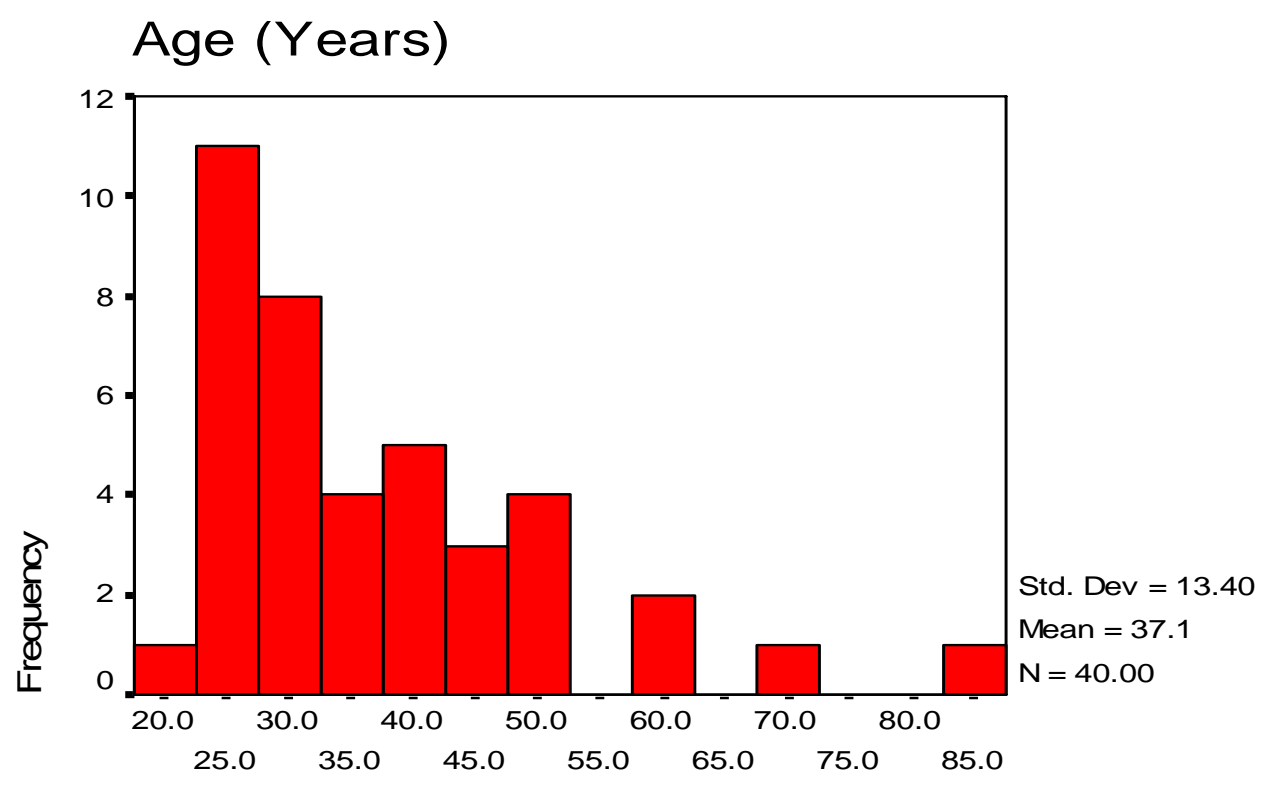

Age (Years) 
Early Management of Open Tibial Fractures in Benin - City-Result of a Structured Treatment Protocol 35

Treatment guidelines for these fractures were followed as closely as possible. These included, initial management at $A \& E$ comprising of full resuscitation and identification of associated injuries, analgesia, splintage, tetanus prophylaxis, wound swabs for microscopy, parenteral antibiotics and sterile wound dressing followed by plain film radiographs. Subsequent management of the injuries was then carried out strictly in casualty theatre as follows;

\section{a. Soft Tissue Management}

i Wound lavage with copious saline and debridement under general anaesthesia, spinal or epidural regional anaesthesia.

ii. Wound classification using Gustillo and Anderson (G - A) system.

寓. Wounds that are made surgically clean were closed Primarily [ (primary closure (PC) ] .

ì. Moderately contaminated dirty wound were left open and closed within 7 - 10 days by Delayed Closure (DC) .

v Heavily contaminated wound and wound defects were left open to heal by secondary intention followed by skin coverage (DSTSG or DFL) .

\section{b. Fracture Management;}

The immediate definitive technique employed for immoilization varied with the associated soft tissue injury and fracture configuration. (Table 2) Plaster of Paris, intemal fixation and external fixation were used where appropriate.

Treatment outcome was analysed on the basis of whether or not primary healing was achieved with each method of treatment. Adjudged successful were cases where the initial method of soft tissue management and fracture fixation resulted in primary healing or eventual healing without resorting to revision surgery. Regarded as failed were cases where healing did not occur primarily and the patient had to undergo one or more revisions to achieve healing. Osseous or fracture healing in this context means union.

\section{Data Analysis}

The results were analysed using the statistics program SPSS Version 12. Significant difference was applied when $\mathrm{P}<0.05$.

\section{Result}

The sex and age distribution is seen in diagrams 1 . The distribution of fractures according to Gustilo were, 2 type I, 17 type II, 9 type III ${ }^{\mathrm{a}}$, 8 type III $^{\mathrm{b}}$ and 3 type IIIc injuries (Table I) . Road traffic accidents contributed collectively over $80 \%$ of the causes of injury (Table II) .

The various methods adopted for primary soft tissue management are shown in Table III. 17 patients had primary closure, 2 of type I, 10 of type II and 5 of type III . Delayed primary closure was performed in 6 cases, 4 type II and 2 type III .16 cases, had delayed skin grafting or flap coverage; 3 in type II, 2 type III 8 type III', 3 in type III'.

In the group treated by primary closure, all type I injuries were successful, while 59\% and 40\% were successful in type II and IIIa injuries respectively. All the cases treated by delayed closure, 4 in type II and 2 in type III healed primarily (100\%) .

Patients treated with the PC method in this study had better results when compared with those whom delayed split thickness skin graft method was used ( $\mathrm{P}=0.013)$.

However, the success rate with delayed skin grafting was high in type II and III injuries and poor with type III and IIIc. There was no significant difference between the success and failure rates ( $\mathrm{P}=0.383 \mathrm{P}>0.05)$, between type IIIa and type III ${ }^{\mathrm{bsc}}$ using skin grafting. Fasciocutanous flap coverage, which were carried out on type III ${ }^{b}$ and III injuries resulted in 50\% failure rate due to poor take as a result of rim necrosis or outright failure of overlying skin in 50\% of the case. P>0.05 $(\mathrm{P}=0.000)$.

Various stabilization methods were employed as primary device. Table VI shows the distribution of stabilization methods used

( C CMS UNIBEN JMBR 2006; 5 (2): 33-39 
Table I: Fracture grading using Gustillo Anderson system in 39 patients

\begin{tabular}{ccc}
\hline Gustilo Grade & No & Percentage \\
\hline I & 2 & 5.1 \\
I & 17 & 43.6 \\
IIla & 9 & 23.1 \\
IIIb & 8 & 20.5 \\
IIIc & 3 & 7.7 \\
\hline Total & 39 & 100 \\
\hline
\end{tabular}

Table II: Aetiology of Injury

\begin{tabular}{|c|c|c|c|}
\hline \multicolumn{2}{|c|}{ Cause } & \multirow{2}{*}{$\frac{\text { Number }}{27}$} & \multirow{2}{*}{$\frac{\text { Percentage }}{65}$} \\
\hline a & Road traffic Accident Automobile (Car) & & \\
\hline & Automobile Bus & 3 & 7.3 \\
\hline $\mathrm{b}$ & motorcycle & 5 & 12.5 \\
\hline C & Fall from height & 1 & 2.4 \\
\hline d. & Gunshot & 3 & 7.3 \\
\hline e & Physical assault & 1 & 2.4 \\
\hline & Unknown & 1 & 2.4 \\
\hline \multicolumn{2}{|c|}{ Total } & 41 & 100 \\
\hline
\end{tabular}

Table III: Soft Tissue Management Methods

\begin{tabular}{llcc}
\hline Type & Number & Percentage \\
\hline a & Primary Closure $\{$ PC $\}$ & 17 & 43.6 \\
b. Delayed Primary Closure $\{$ DPC & 6 & 15.4 \\
c & Delayed Split thickness $\{$ DSTSG & 10 & 25.6 \\
d. Delayed Flaps $\{$ Flaps & 6 & 15.4 \\
\hline
\end{tabular}

Table IV: Stabilization method

\begin{tabular}{llcc}
\hline \multicolumn{1}{c}{ Type } & Number & Percentage \\
\hline a & POP* & 12 & 30.8 \\
bo & IMN** & 13 & 33.3 \\
C & Plating & 6 & 15.4 \\
d & Exofixation & 8 & 20.5 \\
\hline
\end{tabular}

*Plaster of Paris

**Intramedullary Nailing using Kuntscher nails.

and their success rates. The success or failure of these devices to hold till union was largely dependent on soft tissue management outcome. Internal fixation (Intramedullary Nailling or Plating) failure in some cases were due to deep persistent infection causing oteomyelitis and implant loosening. Of the 13 primary K-nailing selectively performed in all the groups 4 failed, 2 in type II and 1 each in type III and type III'. 
Table V: Distribution of Soft Tissue wound closure in each Gustilo type and success rate in the 39 patients

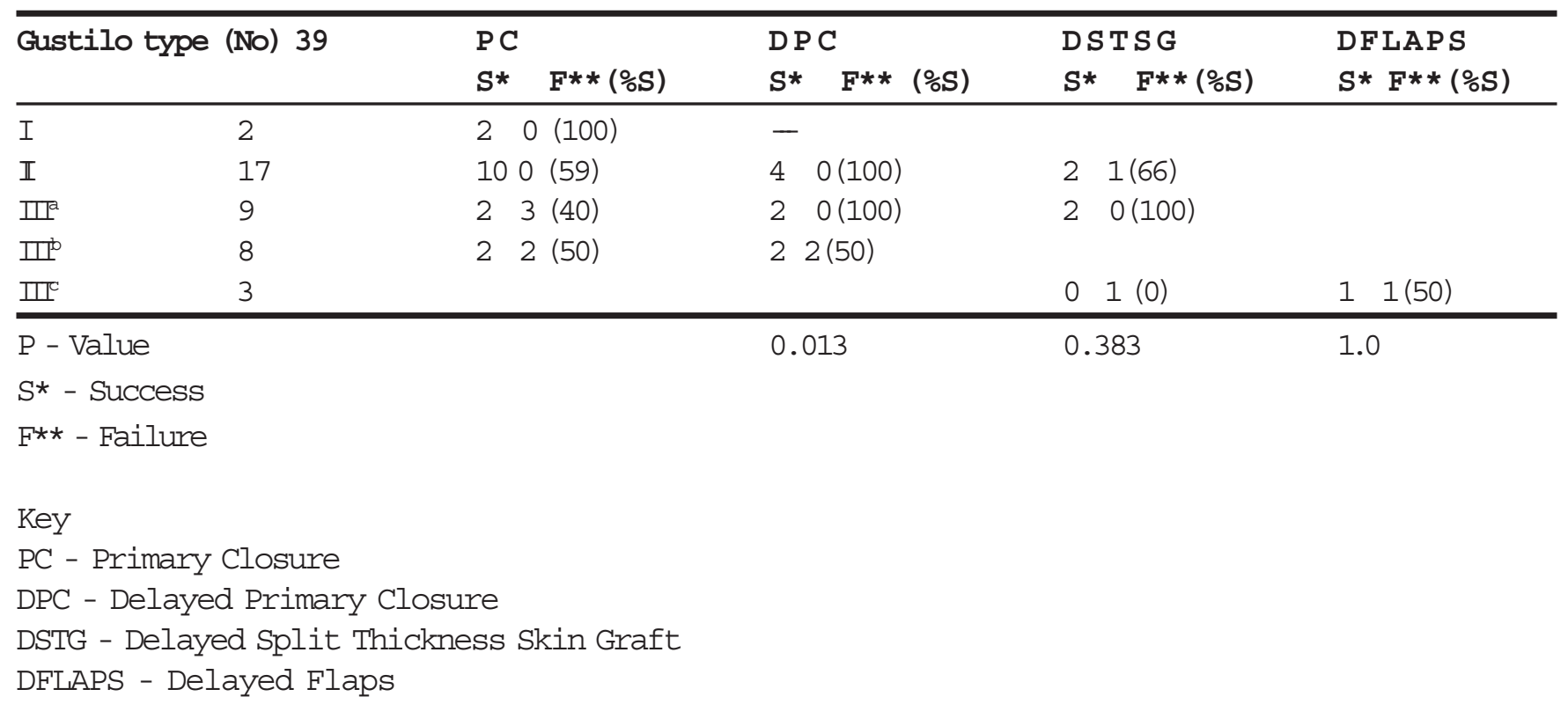

Table VI: Distribution of Stabilization method in each Gustillo type fracture of success rate

\begin{tabular}{|c|c|c|c|c|c|c|c|c|c|}
\hline \multirow{2}{*}{\multicolumn{2}{|c|}{ Gustillo Type (NO.39) }} & \multicolumn{2}{|c|}{ POP } & \multicolumn{2}{|c|}{ K-NAIL } & \multicolumn{2}{|c|}{ Plating } & \multicolumn{2}{|c|}{ Exofixation } \\
\hline & & $\mathrm{S}$ & $\mathbf{F}$ & $\mathrm{S}$ & $\mathbf{F}$ & $\mathrm{S}$ & $\mathrm{F}$ & $\mathrm{S}$ & $\mathbf{F}$ \\
\hline I & 2 & 1 & 0 & 1 & 0 & & & & \\
\hline II & 17 & 10 & 0 & 3 & 2 & 0 & 1 & 1 & 0 \\
\hline iiia & 9 & 1 & 0 & 3 & 1 & 2 & 0 & 2 & 0 \\
\hline مiن & 8 & 2 & 1 & 1 & 1 & 2 & 1 & & \\
\hline iic & & & & & & & & 1 & 2 \\
\hline P - Value & & & & 0.8 & & 0.2 & & 0.1 & \\
\hline
\end{tabular}

All the 12 patients who had primary stabilization with Plaster of Paris had successful outcome.

Exofixation succeeded in less than 50\% in the 3 cases of type III $^{\mathrm{c}}$ and the 3 of III $^{\mathrm{b}}$ respectively.

\section{Discussion}

The therapuetic challenge in the management of open tibial fractures is the unsettling frequency of infection and wound sepsis, which complicate the healing of both soft tissue and bone injury. Modern surgery and antibiotics have still not rid orthopaedic surgery of this scourge. Recent emphasis however, on management of soft tissues in adult open tibial fratures has increased awareness of the importance of prompt and adequate treatment.

Structured guideline, management protocol and strict observance of same have been known to help improve the outcome of management of open fractures ${ }^{4}$. Controversy regarding primary closure of some forms of open fractures of types I, II and infrequently some type III ${ }^{a}$ have been raging on with some authors playing safe by advocating that all open fractures be strictly left open probably because of disastrous consequences of primary coverage of the contaminated wound. 
Many however, are of the opinion that the procedure used by proponents of open wound treatment may not have been performed within the precepts and guideline defined for primary open fracture management in the various studies, hence the high failure rates $^{7,8,9,10}$.

One of the main finding in this study was the encouraging success rate registered with primary closure following a strict protocol guideline; there was 100\% success with type I fractures and 60\% success with type II. The success rate compares favorably with similar reports of primary closures ${ }^{11,12}$ and matches the primary closure success rate recorded in elective orthopaedic surgical wounds ${ }^{13}$. In this study however, some forms of type II and III , recorded only $60 \%$ and $22 \%$ success respectively with primary closure. The failure rate might be due to possible underestimation of the extent of initial injury in terms of contamination, crushing or viability of the structures underlying the apparently small skin wound (of less than $3 \mathrm{~cm}$ ) at time of closure.

All the delayed wound closure of type II and type IIIa ( 6 in number) healed 100\% primarily emphasizing the wisdom in the aphorism when in doubt play safe leave open and close later. All type IIIb and IIIC soft tissue management was treated by open wound principle of dressing with repeated serial debridment for weeks, followed by delayed wound coverage. The failure rate recorded in this series with this type of treatment was as high as $50 \%$. The failure in our opinion, was not unconnected with repeated devastating infections as we strove for weeks to keep the wound clean coupled with desiccation of skin edges and baring bone ends. Wound coverage after very long periods of open dressing was technically more difficult because tissue planes had become obscured and difficult to access. Modern osteoplastic techniques of primary management may be the answer to improving the success rate in types III and IIIc
This protocol liberalized the choice of fracture stabilization method based on the group, fracture configuration and the most suitably available material at the time of treatment. We found great success in the use of plaster of Paris and intramedullary nailing with K-nails as first choice method of stabilization in our primary management because they were cheap, available \& easily adaptable to most fracture configuration. Clearly this study has shown that an organized and standard approach with strict treatment protocol is essentially the key to obtaining the good results in open fracture management. Immediate primary management of some forms of open fractures in this study recorded an overall success rate of $45 \%$ with this protocol, implying that with strict compliance of management guidelines, good surgical judgment and early consultation with plastic surgeons where available, an improved cost-effective outcome of early treatment of open fractures can be achieved.

\section{References}

1 Chapman MW. The use of immediate internal fixation in open fractures in open fractures. Orthop Clin North AM 1980; $11(3): 579-591$.

2 Chapman MW, Mahony M. The role of internal management of open fractures. Clin Orthop 1979; 138: 120 - 131.

3. Gustilo RB, Mercow RL, Templeman D. Current concept review. The management of open fractures. J Bone Joint Surg 1990; 72 (A) : $299-304$.

4. Gustilo RB, Anderson JT. Prevention of infection in the treatment of one thousand and twenty-five open fracture of long bones. Retrospective and prospective analysis. J Bone Joint Surg 1976; 58A: 453 - 458.

5. Onabowale BO, Onuminya JE, Essien IAJ, Ukegbu ND. The management of open tibial shaft fractures: The National Orthopaedic Hospital Experience. Nigeria J Surg 1995; $2(2): 37-42$. 
6. Byrd HS, Spicer TE Cierney G. 3rd: Management of Tibial Fractures Plast. Recons. 1985 Nov; 76 (5) : 719 - 30.

7. Chapman MW. Open fractures. In; Rockwood Jr. CA, Green DP Bucholz RW, Editors. Rockwood and Green's fractures in adults. 3rd edition. Philadelphia: JB Leppincott company, 1991; (1) : 223 - 264

8. Ikem IC. , Oginni LM, Bamgboye EA. Open fractures of the lower limb in Nigeria. International Othopaedics ( SICOT) 2001; 25: $386-388$.

9. Holtom PD, Smith AM. Introduction to adult post traumatic osteomyelitis of the tibia. Clin Orthop 1999; 360: $6-13$.
10. Majumders et al. Management of grade IIIb and IIIc compound fractures of tibia with immediate intemal fixation, early soft tissue cover with muscle flaps presented at British Association of plastic surgeons.

11. Brown C. Henderson S. Moore S. (1996); Surgical treatment of patients with open tibial fractures. AORN Journal. 63 (5) : 875 81, 885 - 96; quiz 899 - 906.

12. Irwin A, Gibson P. Aschcroft P. Open fractures of tibia in children. Injury 1995 26: $21-4$.

13. ChapmanMW, Bray TJ, Spiegel PG. Green SA. Operative Orthopaedics 2nd Ed. J.B Lippincott Company 1993 page $368-9$. 